\title{
Supercurrent and quasiparticle interference between two $d$-wave superconductors coupled by a normal metal or insulator
}

\author{
H. X. Tang, Z. D. Wang, and Jian-Xin Zhu \\ Department of Physics, University of Hong Kong, Pokfulam Road, Hong Kong
}

(Received 28 May 1996)

\begin{abstract}
In the presence of an elastic barrier at both interfaces of a mesoscopic $d$-wave superconductor-normalmetal- $d$-wave superconductor (DND junction), the Josephson current at zero temperature is studied by using a simple matrix method. As a limiting case, the tunneling between two $d$-wave superconductors coupled by a insulator barrier (DID structure) is particularly addressed. The effects of sign change and anisotropic gap structure of the $d_{x^{2}-y^{2}}$ superconductor are carefully considered in the Andreev reflection. The coupling of forward-moving quasiparticles and backward-moving quasiparticles with different pair potentials leads to contrasting Andreev spectra in different motion angle regions, which is specific to our model. Unlike conventional superconducting point-contact junctions, the conduction crossing the Fermi surface plays an important role in determining the critical current. Our theoritical results suggest that the dependence of the critical current on the grain boundary tilt angle provides a clue to identify the pairing symmetry of high- $T_{c}$ superconductors.
\end{abstract} [S0163-1829(96)03741-1]

\section{INTRODUCTION}

To probe the symmetry of pairing states in high- $T_{c}$ superconductors, much effort has been devoted to the physical properties of Josephson junctions with a high- $T_{c}$ superconductor as one bank. ${ }^{1,2}$ Although divergent views on the pairing symmetry remain, most theoretical studies proposed that the superconducting states of these materials could be characterized by a $d_{x^{2}-y^{2}}$ symmetry. Other candidates are $s$-wave, anisotropic $s$-wave, or a mixed $s+i d$-wave state. It has been known that a $d$-wave superconductor not only has a vanishing order parameter for certain directions of the Fermi surface but also changes its sign, with a function of $k_{a}^{2}-k_{b}^{2}$. On this line, a variety of experimental investigations in connection with the Josephson effect have been developed in the past several years toward the identification of the symmetry of the order parameter in high- $T_{c}$ superconductors. Although there remain a few significant measurements that cannot be explained by a pure $d$-wave state, ${ }^{3,4}$ there does exist growing evidence for $d$-wave symmetry in the pair potential of high- $T_{c}$ superconductors. $^{5-9}$

In an earlier report, ${ }^{10} \mathrm{Hu}$ studied the coupling of oppositesign order parameter quasiparticles, and found that there exist midgap states which are definitely absent in any type of $s$-wave superconductor junctions. Very recently, Xu, Miller, and Ting investigated the effects of Andreev reflection on the current-voltage characteristic and differential conductance of a normal metal and a $d$-wave superconductor. ${ }^{11}$ Considering the coupling between the direction-sensitive order parameter of two linked conductors, the frequently observed zero-bias conductance peaks (ZBCP's) and gaplike conductance spectra between a normal metal and a cuprate superconductor were attributed to the interface bound states originating from the assumed $d$-wave gap in one superconducting bank. ${ }^{11-13}$ The barrier potential plays an important role in the formation of these bound states and is essential to the ZBCP's. The Josephson coupling between a high- $T_{c}$ superconductor and a low $T_{c}$ superconductor has been shown to be effective in discriminating the paring state, both experimentally ${ }^{5-7}$ and theoretically. ${ }^{14,15}$ The paring symmetry can also demonstrate itself from a critical supercurrent of a junction composed of relatively tilted $d$-wave superconductors (or grain boundary). ${ }^{4}$ In the presence of a dielectric barrier at the interface, the transport along a direction other than normal to the interface was thought to be negligible, which leads to the critical current ${ }^{16}$

$$
I_{c}=A \cos 2 \alpha_{1} \cos 2 \alpha_{2},
$$

where $A$ is a constant and $\alpha_{1}$ and $\alpha_{2}$ are the relative angles of the two superconductors to the orientation of the grain boundary face. In a special device with $\alpha_{1}=\alpha_{2}=\alpha-$ i.e., the crystal axis orientations of two $d$-wave superconductors coincide-the variation of the critical current is reduced to $A(\cos 2 \alpha)^{2}$. It follows that no supercurrent will flow through the junction for a $\{110\}$ grain boundary. But as figured in Refs. 10-12, when the coupling of quasiparticles of two directions with an opposite-sign order parameter happens, whether it comes from a surface or an insulating barrier, midgap states or bound states appear and lead to an enhanced conductance. In the same way, for two superconductors, the Josephson current will be greatly influenced by the barrier at the interface between them. In fact, we will show later on in this paper, at the special angle $\alpha=45^{\circ}$, in most cases, the proposed midgaps would carry a maximum supercurrent, not zero. In addition, the barrier at the interface will give rise to the considerable continuum current carried by the quasiparticles, which was not taken into account in Eq. (1) and disappears in conventional superconducting point-contact junctions. ${ }^{17}$

When there is no barrier at the interface, or Sharvin metallic contacts, obviously the direction normal to the interface no longer holds priority over others. A complete theory should include all these directions. Owing to the development of nanofabrication technology, clean-limit super- 


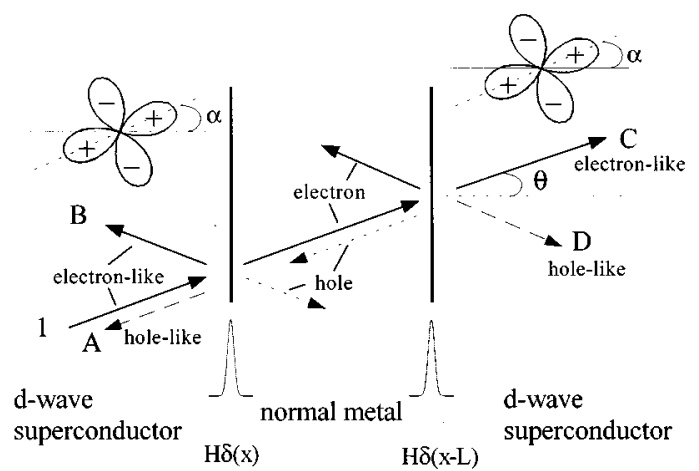

FIG. 1. The DND system we studied consists of two $d$-wave superconductors with concident crystal oriention linked by a normal conductor with length $L$. An electronlike excitation incident from the left electrode is reflected as electronlike and holelike quasiparticles and transmitted to the right contact as both type quasiparticles. The pair potential $\Delta(x, \theta)$ is a function of $\theta$. The insulating barrier is assumed to form a spacial potential $H \delta(x)+H \delta(x-L)$.

conductor-normal-metal-superconductor $(S N S)$ heterostructure is realized ${ }^{18}$ and quantum transport is found to take effect. The nature of the paring state will be more pronounced once this technique is applied to high- $T_{c}$ superconductors. In this paper, we propose a simple topology to test for interference effects between two $d$-wave superconductors and hence for the pairing symmetry. Based on the Andreev approximation, a simple matrix method is developed to derive the transmission matrix of the model DND superconducting structure in Sec II. In Sec III, the phase-dependent supercurrent of metallic $d$-wave superconductor-normalmetal- $d$-wave superconductor (DND) structure both in short and long limits is calculated. The tunneling current through a superconducting point conduct with insulating interface barrier is particularly addressed in Sec IV. Many anomalous characteristics of our configuration are exposed. Finally, a brief summary is given.

\section{MODEL AND TRANSMISSION MATRIX}

Let us consider a system consisting of two twodimensional $d$-wave superconductors coupled by a normal metal of length $L$, which is schematically drawn in Fig. 1. At the interfaces of both $N S$ junctions, the tunneling barrier has a delta-function form $H \delta(x)$. In the following, we will simply neglect the Fermi velocity mismatch effect, which is said to be equivalent to an effective enhanced barrier at the interface. ${ }^{19}$ As usual, by ignoring the proximity effects, we adopt the steplike superconducting pair potential

$$
\Delta(x, \theta)= \begin{cases}\Delta_{0} \cos (2 \alpha-2 \theta) e^{-i \phi / 2}, & x<0, \\ 0, \quad 0 \leqslant x \leqslant L, & \\ \Delta_{0} \cos (2 \alpha-2 \theta) e^{i \phi / 2}, & x>L,\end{cases}
$$

where $\theta$ is the quasiparticle motion direction with reference to the orientation of the junction. The phase difference across the junction is $\phi$. As exhibited in Fig. 1, due to the existence of normal reflection at the interface, an injected electronlike quasiparticle is reflected as both electronlike and holelike quasiparticles. To keep the conservation of both the momen- tum parallel to the interface and the group velocity at the interface, the reflected electronlike quasiparticle moves in the direction $\pi-\theta$, experiencing a pair potential $\Delta(-\theta)$ in this $d$-wave superconductor. The same thing occurs at the right $N S$ interface: The transmitted electronlike and holelike quasiparticles are subjected to two different pair potentials $\Delta(\theta)$ and $\Delta(-\theta)$, respectively. In our assumption of identical sphere Fermi surfaces, on crossing the interface, electrons propagate at an unchanged angle; namely, no refraction takes place. In the middle normal conductor, all four kinds of conducting particles are coupled: right- and left-going electrons $\left(\Psi_{e}^{ \pm} \propto e^{ \pm i k_{x}^{+} x+i k_{y} y}\right)$ and holes $\left(\Psi_{h}^{\mp} \propto e^{\mp i k_{x}^{-} x+i k_{y} y}\right)$, where $k_{x}^{ \pm}=k_{F} \cos (\theta)\left[1 \pm E / E_{F} \cos ^{2}(\theta)\right], k_{y}=k_{F} \sin (\theta)$. In the Andreev approximation, matching the wave functions across the right $N S$ junction with an elastic barrier $H$, we obtain the following coefficients for an injected electron with angle $\theta$ :

$$
\begin{gathered}
a_{+}=u(-\theta) v(\theta) / \gamma, \\
b_{+}=[u(-\theta) u(\theta)-v(-\theta) v(\theta)]\left[-i Z(\theta)-Z(\theta)^{2}\right] / \gamma, \\
c_{+}=u(-\theta)[1-i Z(\theta)] / \gamma, \\
d_{+}=v(\theta) i Z(\theta) / \gamma,
\end{gathered}
$$

where $Z(\theta)=H / \hbar v_{F} \cos (\theta)=Z / \cos (\theta), \quad \gamma \quad$ is defined as $u(\theta) u(-\theta)\left[Z(\theta)^{2}+1\right]-v(\theta) v(-\theta) Z(\theta)^{2}$ and $u(\theta)$ and $v(\theta)$ represent the BCS coherence factors,

$$
\begin{gathered}
u(\theta)=\left(\frac{1}{2}+\frac{1}{2} \sqrt{\frac{\Delta(\theta)^{2}-\epsilon(\theta)^{2}}{\epsilon(\theta)^{2}}}\right)^{1 / 2}, \\
v(\theta)=\left(\frac{1}{2}-\frac{1}{2} \sqrt{\frac{\Delta(\theta)^{2}-\epsilon(\theta)^{2}}{\epsilon(\theta)^{2}}}\right)^{1 / 2} \operatorname{sgn}[\Delta(\theta)] .
\end{gathered}
$$

Those four coefficients in Eqs. (3) correspond to the probability amplitudes for Andreev reflection, normal reflection, transmission without crossing Fermi surface, and transmission crossing Fermi surface. They are $\theta$ dependent not only in that the order parameters are direction-sensitive but also in the directly $\theta$-dependent effective barrier strength. In the case of hole injection, a substitution of $\theta$ by $-\theta$ and $Z$ by $-Z$ will give the corresponding coefficients $a_{-}, b_{-}, c_{-}, d_{-}$, respectively. Then we can use the following two matrices to describe the reflected electron-hole wave pack $\left(\Psi_{e}^{+}, \Psi_{h}^{-}\right)$and transmitted quasiparticle wave pack with respect to the injected wave pack $\left(\Psi_{e}^{-}, \Psi_{h}^{+}\right)$:

$\hat{r}(\theta)=\left(\begin{array}{ll}b_{+}(\theta) & a_{-}(\theta) \\ a_{+}(\theta) & b_{-}(\theta)\end{array}\right), \quad \hat{O}(\theta)=\left(\begin{array}{cc}c_{+}(\theta) & d_{-}(\theta) \\ d_{+}(\theta) & c_{-}(\theta)\end{array}\right)$.

Taking the phase factor $\phi$ into account, at the right NS junction, we can write

$$
\begin{gathered}
\hat{r}(\theta, \phi / 2)=\hat{\pi}(\phi / 2) \hat{r}(\theta) \hat{\pi}(-\phi / 2), \\
\hat{O}(\theta, \phi / 2)=\hat{O}(\theta) \hat{\pi}(-\phi / 2),
\end{gathered}
$$

with

$$
\hat{\pi}(\phi / 2)=\left(\begin{array}{cc}
e^{i \phi / 4} & 0 \\
0 & e^{-i \phi / 4}
\end{array}\right) .
$$


If we track the reflected wave pack, one can see that after freely evolving to the left interface, it is reflected and then returns to the right interface.

At the left $S N$ interface, we use a matrix $\hat{I}(\theta,-\phi / 2)$ to denote the transformation of the quasiparticle in the left bank into the electrons and holes in the normal conductor. In a similar matrix form

$$
\hat{I}(\theta,-\phi / 2)=\hat{\pi}(-\phi / 2) \hat{I}(\theta)=\hat{\pi}(-\phi / 2)\left(\begin{array}{cc}
c_{+}^{\prime}(\theta) & d_{-}^{\prime}(\theta) \\
d_{+}^{\prime}(\theta) & c_{-}^{\prime}(\theta)
\end{array}\right) .
$$

For an electronlike quasiparticle injected from the $S$ side, the amplitudes of electrons and holes feed into the $N$ side, $c_{+}^{\prime}, d_{+}^{\prime}$ are found to be related to $c_{+}$and $d_{-}$,

$$
\begin{aligned}
& c_{+}^{\prime}(\theta)=c_{+}(\theta)\left[u^{2}(\theta)-v^{2}(\theta)\right], \\
& d_{+}^{\prime}(\theta)=d_{-}(\theta)\left[u^{2}(\theta)-v^{2}(\theta)\right] .
\end{aligned}
$$

The corresponding amplitude coefficients in the case of holelike quasiparticle injection can be deduced similarly,

$$
\begin{aligned}
& c_{-}^{\prime}(\theta)=c_{-}(\theta)\left[u^{2}(-\theta)-v^{2}(-\theta)\right], \\
& d_{-}^{\prime}(\theta)=d_{+}(\theta)\left[u^{2}(-\theta)-v^{2}(-\theta)\right] .
\end{aligned}
$$

The matrix corresponding to one round successive processes $\left(L_{\rightarrow}^{\hat{\tau}} R+\right.$ reflection, $\stackrel{\hat{\tau}}{\rightarrow} \rightarrow L+$ reflection $)$ can be worked out,

$$
\begin{aligned}
\hat{M}(\theta, \phi) & =\hat{r}(\pi-\theta,-\phi / 2) \hat{\tau} \hat{r}(\theta, \phi / 2) \hat{\tau} \\
& =\hat{r}(-\theta,-\phi / 2) \hat{\tau} \hat{r}(\theta, \phi / 2) \hat{\tau}
\end{aligned}
$$

where $\hat{\tau}$ is the free propagation matrix,

$$
\hat{\tau}=\left(\begin{array}{cc}
e^{i k_{x}^{+} L} & 0 \\
0 & e^{-i k_{x}^{-} L}
\end{array}\right)
$$

The overall transmission matrix can be calculated in view that a charge-carrying electronlike (or holelike) quasiparticle is injected from the left superconducting bank, producing electron and holes in the middle conductor; after multiple reflection processes, these electrons and holes escape to the right superconductor in the form of electronlike and holelike quasiparticles. It follows that

$$
\hat{T}(\theta, \phi)=\hat{O}(\theta, \phi / 2) \hat{\tau}[1-\hat{M}(\theta, \phi)]^{-1} \hat{I}(\theta,-\phi / 2) .
$$

Here we use $\hat{T}(\theta, \phi)$ to denote the overall transmission matrix of the superconducting device at a specific angle.

The discrete energy levels in the DND pair potential can be determined by the poles of the transmission matrix,

$$
\Gamma(\theta, \phi)=\operatorname{det}[1-\hat{M}(\theta, \phi)]=0 .
$$

The resulting energy levels $E_{n}(\theta, \phi)$ in the superconducting gap are responsible for the discrete part of supercurrent. On the other hand, the continuum current carried by the quasiparticles out of the energy gap can be computed from the transmission matrix.

\section{SUPERCURRENT THROUGH A CLEAN DND HETEROSTRUTURE}

As the first step, we shall investigate the supercurrent in this model device with no barrier potential at the grain boundary. Since the basic assumption that the transport in the vicinity of the junction direction dominates the current is no longer valid, the solution is complicated by the anisotropic energy gap and the different phase-coherent paths in the normal region. In the absence of normal reflection at the boundary, the right-going electron is reflected back only in the form of a hole in the inverse direction, and no gap change can be seen by it. The diagonal transfer matrix is simplified as

$$
\hat{T}(\theta, \phi)=\left(\begin{array}{cc}
\frac{e^{-i \phi / 2} e^{i k_{F} \cos (\theta) L}\left(1-\left[v^{2}(\theta) / u^{2}(\theta)\right]\right)}{1-\left[v^{2}(\theta) / u^{2}(\theta)\right] e^{-i \phi+i(\epsilon / \Delta)[L / \xi \cos (\theta)]}} & 0 \\
0 & \frac{e^{i \phi / 2} e^{-i k_{F} \cos (\theta) L}\left(1-\left[v^{2}(-\theta) / u^{2}(-\theta)\right]\right)}{1-\left[v^{2}(-\theta) / u^{2}(-\theta)\right] e^{i \phi+i(\epsilon / \Delta)[L / \xi \cos (\theta)]}}
\end{array}\right) .
$$

The element $\hat{T}_{11}$ represents the amplitude for an electronlike quasiparticle transmitted into the right electrode without crossing the Fermi surface, while $\hat{T}_{22}$ is the exact counterpart for holelike quasiparticle injection. No process with quasiparticle crossing the Fermi surface is involved. At the angle $\theta$, the poles of $\hat{T}_{11}$ and $\hat{T}_{22}$ shapes the energy spectrum,

$$
2 \cos ^{-1} \frac{\epsilon_{n \pm}(\theta, \phi)}{|\Delta( \pm \theta)|} \pm \phi-\frac{\epsilon_{n \pm}(\theta, \phi)}{\Delta_{0}} \frac{L}{\xi \cos (\theta)}=2 n \pi .
$$

Here $\Delta( \pm \theta)$ stand for the $d$-wave gaps at $\theta$ and $\pi$ - $\theta$ orientations. At zero temperature, the phase-difference induced supercurrent carried by these Andreev levels can be identified as

$$
I_{d}(\theta, \phi)=\sum_{\epsilon<0, n} \frac{2 e}{\hbar} \frac{d \epsilon_{n \pm}(\theta, \phi)}{d \phi} .
$$

For a junction with a finite length, there exist leaky states out of the superconducting gap or, say, current-carrying continuum spectrum. As the holelike branch is equivalent to the 

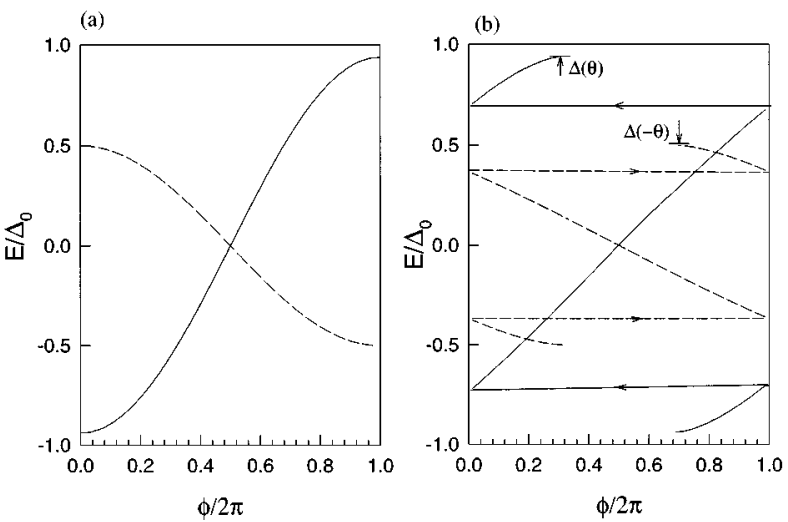

FIG. 2. (a) The Andreev levels in a point contact junction with $L=0$. (b) Long junction with $L=2 \xi_{0}$. Solid lines represent the positive process while the dashed line the negative process.

electronlike branch in carrying the continuum supercurrent, ${ }^{17}$ in the following, we treat only the electron branch. At a specific angle, accounting for the backflow of supercurrent in the zero-temperature equilibrium state, we find that the electrical current carried by electronlike excitations in the continuum spectrum is

$$
\begin{aligned}
I_{c}(\theta, \phi)= & \frac{2 e}{h} \int_{-\infty}^{-\left.|\Delta(\theta)||| \hat{T}_{11}(\theta, \phi)\right|^{2}-\left|\hat{T}_{11}(\theta,-\phi)\right|^{2}} \\
= & \frac{2 e}{h} \int_{-\infty}^{-|\Delta(\theta)|}\left|u^{2}(\theta)-v^{2}(\theta)\right| \\
& \times\left[\frac{1}{D(\theta, \phi)}-\frac{1}{D(\theta,-\phi)}\right] d \epsilon,
\end{aligned}
$$

where $\quad D(\theta, \phi)=u^{4}(\theta)+v^{4}(\theta)-2 u^{2}(\theta) v^{2}(\theta) \cos [\phi-\epsilon L /$ $\left.\xi \Delta_{0} \cos (\theta)\right]$. Our calculation on the supercurrent will be carried out in short and long junction limits. For a zero-length metallic point contact, Eq. (16) reduces to the asymmetric Andreev levels [Fig. 2(a)],

$$
\begin{gathered}
\epsilon_{+}(\theta)=-\Delta_{0}|\cos (2 \alpha-2 \theta)| \cos \left(\frac{\phi}{2}\right), \\
\epsilon_{-}(\theta)=\Delta_{0}|\cos (2 \alpha+2 \theta)| \cos \left(\frac{\phi}{2}\right) .
\end{gathered}
$$

The integration of the supercurrent along all directions results in the total discrete current being independent of the crystal orientation,

$$
I_{d}(\phi)=\frac{2 e \Delta_{0}}{\hbar} \sin \left(\frac{\phi}{2}\right) \operatorname{sgn}(\phi-\pi) .
$$

Apparently, in the absence of leaky states above the gap, no continuum current flows through the junction when $L=0$. Thus the critical current for a metallic point contact between two parallel $d$ waves shows isotropic behavior just like $s$-wave superconductors, rather than obeying Eq. (1), as a result of averaging over the contributions of all directions. Despite its asymmetric feature along one specific direction, the symmetric phase dependence of the total Josephson current is preserved if we incorporate conduction along the
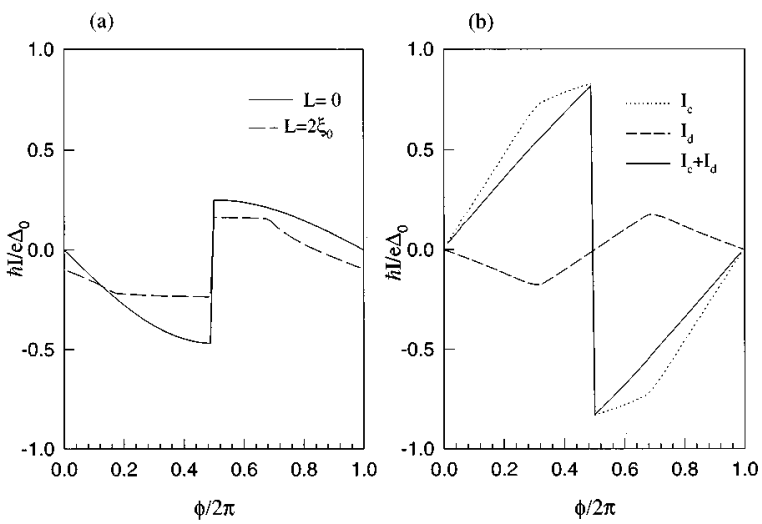

FIG. 3. (a) Discrete current corresponding to the Andreev levels in Fig. 2. (b) The discrete current $I_{d}$ (dashed line), continuum current $I_{c}$ and total Josephson current (solid line) for a junction with $L=2 \xi_{0}, \alpha=20^{\circ}$.

$\pi$ - $\theta$ direction. When the junction length $L$ becomes comparable to $\xi$, the number of Andreev levels within the energy gap grows and increases with the conduction angle. The discrete Andreev spectrum for a junction with $L=2 \xi, \alpha=20^{\circ}$ is shown in Fig. 2(b). The discrete currents corresponding to a particular energy spectrum in Fig. 2(b) are graphed in Fig. 3 (a) as solid lines. For a nonzero-length junction, the continuum levels begin to carry a finite supercurrent. After integrating both the discrete and continuum current denotions of all angles, a reduced total supercurrent for a junction with $L=2 \xi$ is found as compared with the point-contact junction [Fig. 3(b)]. The triangular dependence of the supercurrent on the phase in the long junction limit ${ }^{22}$ is obtained.

We now proceed to calculate the dependence of the critical current on the junction inclination angle, which will certainly display the anisotropic nature of $d$-wave superconductors. By summing the continuum and discrete currents, the critical currents of DND structures for four different values of junction length are computed, as depicted in Fig. 4. The

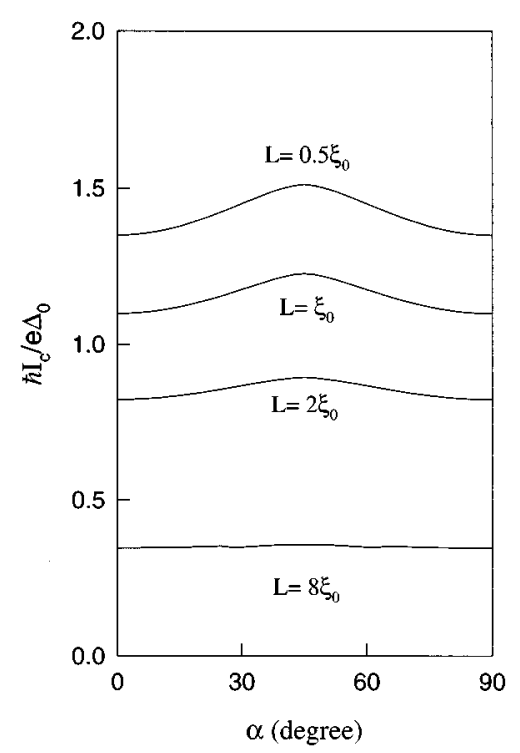

FIG. 4. The orientation angle dependence of critical current of a DND structure for four different junction lengths. 
variation of the critical supercurrent shows an increase tendency when the grain boundary approaches the $\{110\}$ surface. In addition to a reduced overall supercurrent, the cusplike curve is flattened when the normal metal width is increased from $0.5 \xi$ to $8 \xi$. In the case of a point contact, the critical current has been shown to be a constant value $2 e \Delta_{0} / \hbar$, being independent of the angle $\alpha$. The critical current in the long junction limit $(L>8 \xi)$ shows the same trend but with a far reduced magnitude. The critical current of $S N S$ junctions consisting of both isotropic $s$-wave superconducting banks is in no way dependent on the tilt angle, but an anisotropic $s$-wave or a mixed-wave gap model may give a similar effect. The consequence of the sign change of the $d$-wave superconducting order parameter is not exhibited in our metallic normal barrier junctions.

\section{SUPERCURRENT THROUGH A $d$-WAVE SUPERCONDUCTING POINT CONTACT}

As stated in the Introduction of this paper, the motivation for this work comes from the anomalous subgap structure in a $d$-wave superconductor-normal-metal point contact, which was supposed to be the origin of the observed subgap con- ductance enhancement. ${ }^{10-12}$ In this section, we concentrate on the barrier effect on the supercurrent flowing across a junction shorter than the healing length $(L<\xi)$, with contributions from all possible directions properly included. In this limit, we are actually dealing with a DID structure with the insulating barrier strength $Z^{*}=2 Z$. Writing the transmission matrix corresponding to Eq. (13) in the form

$$
\hat{T}(\theta, \phi)=\left(\begin{array}{ll}
C(\theta, \phi) & D^{*}(\theta, \phi) \\
D(\theta, \phi) & C^{*}(\theta, \phi)
\end{array}\right),
$$

we work out the elements $C(\theta, \phi), D(\theta, \phi)$ representing electronlike and holelike components formed in the right electrode for an electronlike quasiparticle injection,

$$
\begin{aligned}
C(\theta, \phi)= & \left(1+i Z^{*}\right) e^{-i \phi / 2}\left[1-v(\theta)^{2} / u(\theta)^{2}\right]\left[1-e^{i \phi} v\right. \\
& \left.(-\theta)^{2} / u(-\theta)^{2}\right] / \Gamma(\theta, \phi), \\
D(\theta, \phi)= & -i Z^{*} e^{-i \phi / 2}\left[1-v(\theta)^{2} / u(\theta)^{2}\right][u(-\theta) v(\theta) \\
& \left.-e^{i \phi} u(\theta) v(-\theta)\right] / u(-\theta)^{2} / \Gamma(\theta, \phi),
\end{aligned}
$$

with

$$
\Gamma(\theta, \phi)=\frac{\left[-v^{2}(\theta) / u^{2}(\theta)\right] e^{-i \phi}-\left[v^{2}(-\theta) / u^{2}(-\theta)\right] e^{i \phi}+\left(1+Z^{* 2}\right)\left\{1+\left[v^{2}(\theta) v^{2}(-\theta) / u^{2}(\theta) u^{2}(-\theta)\right]\right\}-2 Z^{* 2}[v(\theta) v(-\theta) / u(\theta) u(-\theta)]}{\left\{Z^{2}+1-Z^{2}[v(\theta) v(-\theta) / u(\theta) u(-\theta)]\right\}^{2}} .
$$

The numerator, which gives the energy spectrum of the DID system, in the Sharvin metallic point-contact limit, i.e., $Z^{*}=0$, can be factored into two decoupling parts,

$$
\Gamma(\theta, \phi)=\left[1-\frac{v^{2}(\theta)}{u^{2}(\theta)} e^{-i \phi}\right]\left[1-\frac{v^{2}(-\theta)}{u^{2}(-\theta)} e^{i \phi}\right]
$$

leading to asymmetric right- and left-going energy spectra. Notice that in Eq. (25), at all angles, only the absolute values of order parameters take effect instead of a $\pi$-phase shift involved in a $d / s$ or $d / n$ contact. ${ }^{10-12}$ As pointed out in Sec. III, these two waves transport independently just like a usual clean SNS device. Apart from a fourfold symmetry modulus, the sign change of the order parameter is not manifested. To understand this usual Andreev process, the relevant quasiparticle trajectories are shown in the upper part of Fig. 5. The right-moving quasiparticle confined by the pair potential $\Delta(\theta)$ couples with its time-reversed quasiparticle and forms an Andreev state of size $\xi$ bound near the interface, with the left-moving bound state confined by $\Delta(\theta)$. The lower part of Fig. 5 implies that the right-moving state is coupled through the scattering barrier to the left-moving state; both $\Delta(\theta)$ and $\Delta(-\theta)$ play a role in the formation of quasibound states. From Eqs. (22), (23), and (24), it can be learned that a finite barrier strength $Z$ mixes $v(\theta)$ and $v(-\theta)$, not only giving a distinctive feature to the energy spectrum, but also raising the transport probability across the Fermi surface. In Eq. (22), it is $|\Delta(\theta)|$ that responds for the electronlike quasiparticle conduction, while as implied in Eq. (23), the supercurrent contribution from the holelike quasiparticles created at the right superconducting bank will show a sense of $\pi$ shift when the gap signs of opposite directions are reversed. The continuum current carried by this branch shall be much more pronounced.

In a more compact formula, the Andreev energy levels are determined by

$$
\cos \left(\phi-\vartheta_{+}+\vartheta_{-}\right)=\left(1+Z^{* 2}\right) \cos \left(\vartheta_{+}+\vartheta_{-}\right)-Z^{* 2} .
$$

Here, $\quad \vartheta_{ \pm}=\arccos \left(\epsilon / \Delta_{ \pm}\right) \quad$ while $\arccos (t) \equiv-i \ln \left[1+\left(t^{2}\right.\right.$ $\left.-1)^{1 / 2}\right]$ for $t>1$. Two quasibound states are formed when $|\epsilon|<\min [|\Delta(\theta)|,|\Delta(-\theta)|]$. Fixing $\alpha$ at $20^{\circ}$, the Andreev levels $\epsilon_{ \pm}(\phi)$ are plotted in Fig. 6 as a function phase difference $\phi$. As expected, when $\theta=20^{\circ}$, the signs of the two related order parameters are unique; the curves are not only flattened but shifted due to the existence of a finite $Z$ value. The interface barrier opens an energy gap in the Andreev spectrum versus the superconducting phase difference, consequently suppressing the supercurrent. While when the quasiparticle moving direction deviates from the normal angle and consequently leads to an opposite sign in the pair potentials, the gap in the energy spectrum vanishes with the corresponding nodal point located at $\phi=\pi$ just like that in a metallic SNS ( $s$-wave) superconducting point contact, and the supercurrent carried through these angles is significantly enhanced. On the other hand, when $|\epsilon|>\max [|\Delta(\theta)|,|\Delta(-\theta)|]$, it is the continuum spectrum that accounts for the conduction of supercurrents, and no bound states could exist. In the case that energy $\epsilon$ falls between these two gaps, as we mentioned above, in the zero barrier limit, when $|\Delta(\theta)|<|\Delta(-\theta)|$, there is a single bound state with energy $|\Delta(-\theta)| \cos (\phi / 2)$. 

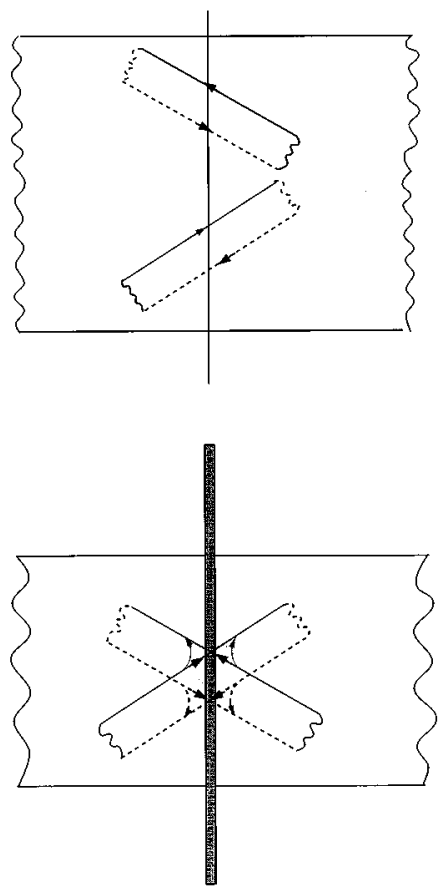
levels.

FIG. 5. The quasiparticle trajectories help to form Andreev

Once the potential barrier applies, the mixing of opposite moving electrons makes this bound state incompletely constrained in the interface by the pair potential or getting "leaky," rather than a long life bound one. It is drawn as an extension of the bound Andreev levels in Fig. 5(a). Particularly, at one special angle, $\Delta(-\theta)$ approaches zero and the energy eigenvalue can be analytically obtained,

$$
\begin{gathered}
\epsilon_{+}=-\frac{|\Delta(\theta)|}{2}\left(\sqrt{1+Z^{* 2}}+\frac{1}{\sqrt{1+Z^{* 2}}}\right) \cos \left(\frac{\phi}{2}\right) \\
-i \frac{|\Delta(\theta)|}{2}\left(\sqrt{1+Z^{* 2}}-\frac{1}{\sqrt{1+Z^{* 2}}}\right) \sin \left(\frac{\phi}{2}\right), \\
\epsilon_{-}=0,
\end{gathered}
$$

which is graphed in Fig. 6(b) with the shadow area expressing the finite lifetime of the eigenstates.

We use Eq. (17) to calculate the discrete current. The phase-dependent supercurrent for our four typical motion angles is plotted in Fig. 7(a). In the $\theta=0$ direction, the forward- and backward-moving electrons are acted on by an unchanged pair potential at the interfaces; its symmetric spectrum carries a nearly sinuoidal supercurrent. When the moving angle tilts to a higher value, part of the discrete current is redistributed to half-bounded states. The interference of quasiparticles with two pair potentials results in a distorted $I(\phi)$ relationship. In the angle region $\pi / 4-\alpha<\theta<\pi / 4+\alpha$, the pair potentials $\Delta(\theta)$ and $\Delta(-\theta)$ have opposite signs and thus there is a sharp discontinuity at $\phi=\pi$. The reported subgap states ${ }^{10-12}$ have a similar origin. These characteristics of the discrete Andreev levels and discrete current are peculiar to the $d$-wave superconducting in-
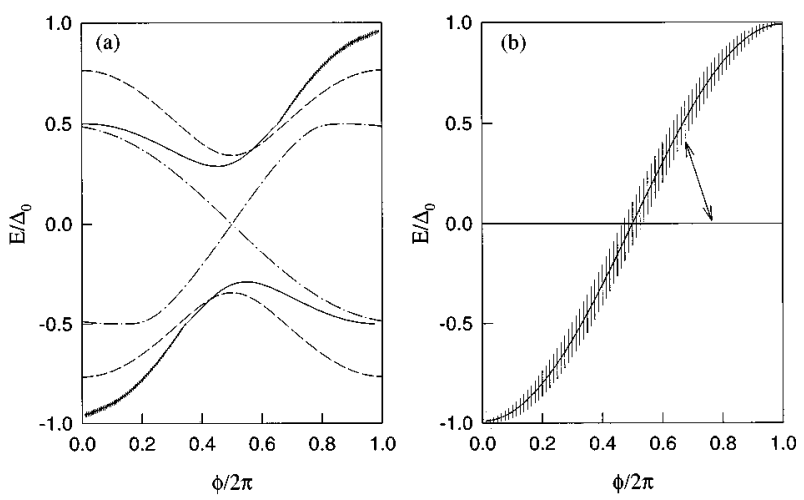

FIG. 6. (a) The Andreev levels spectrum of point-contact DID junction with $Z=0.5, \alpha=20^{\circ}$ at various motion angles: $\theta=0^{\circ}$ (dashed line), $\theta=10^{\circ}$ (solid line), and $\theta=45^{\circ}$ (dot-dashed line). (b) When $\Delta(-\theta)$ approches zero, the negative branch becomes zero, while the positive one gets finite lifetime.

terference topology we studied, being quite different from the results of well-understood SNS heterostructure. ${ }^{17,20,21}$

To compute the current carried by the continuum and half-bound states, we start from the coefficients expressed by Eqs. (22) and (23). Continuum supercurrents are the results of an imbalance of particle transmission from left to right and transmission from right to left, i.e.,

$$
I_{c}(\phi, \theta)=I_{L \rightarrow R}^{e}(\phi, \theta)-I_{R \rightarrow L}^{e}(\phi, \theta) .
$$

In the angle range $|\Delta(\theta)|>|\Delta(-\theta)|$, as the electronlike branch is involved, at zero temperature only quasiparticles with energy smaller than $-|\Delta(\theta)|$ can exist and conduct current to the right electrode as positively propagating electronlike and holelike quasiparticles,

$$
\begin{aligned}
I_{L \rightarrow R}^{e}(\theta, \phi)= & \frac{2 e}{h} \int_{-\infty}^{-|\Delta(\theta)|} \frac{1}{\left|u(\theta)^{2}-v(\theta)^{2}\right|} \\
& \times\left[|C(\theta, \phi)|^{2}-|D(\theta, \phi)|^{2}\right] d \epsilon .
\end{aligned}
$$

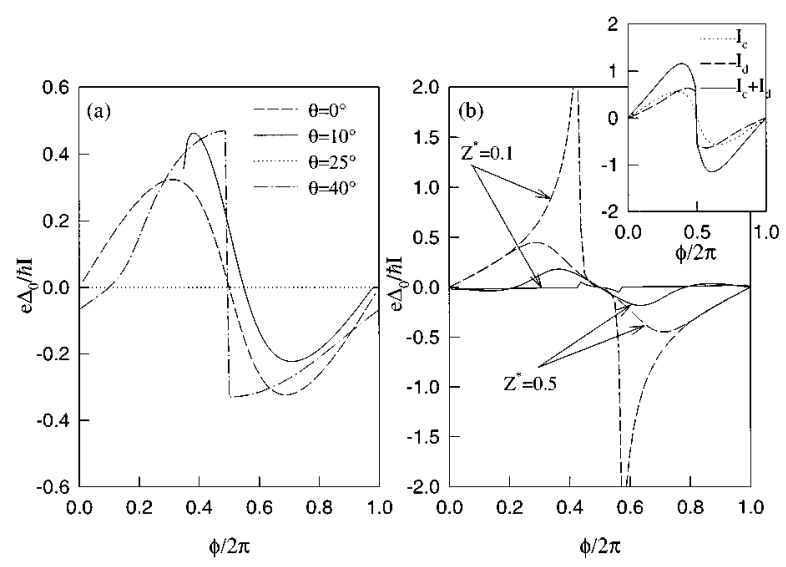

FIG. 7. (a) Calculated discrete supercurrent carried by Andreev levels in Fig. 6. (b) Continuum current through semileaky states. The trasported currents crossing the Fermi surface are plotted as Dashed line show resonant structure for $Z^{*}=0.1$. Inset: an integration of conduction in all directions for $Z^{*}=0.5, \alpha=20^{\circ}$. 
Here the prefactor $1 /\left|u(\theta)^{2}-v(\theta)^{2}\right|$ incorporates the superconducting density of states for quasiparticles incident in this direction. When $|\Delta(\theta)|$ becomes smaller than $|\Delta(-\theta)|$, in the energy range $-|\Delta(-\theta)|<\epsilon<-|\Delta(\theta)|$, free holelike quasiparticles cannot exist in the right electrode. They have a finite lifetime and dissipate into Cooper pairs away from the interface. The continuum currents in this case take the form

$$
\begin{gathered}
I_{L \rightarrow R}^{e}(\theta, \phi)=\frac{2 e}{h} \int_{-\infty}^{-|\Delta(-\theta)|} \frac{1}{\left|u(\theta)^{2}-v(\theta)^{2}\right|} \\
\times\left[|C(\theta, \phi)|^{2}-|D(\theta, \phi)|^{2}\right] d \epsilon \\
\text { for } \epsilon<-|\Delta(-\theta)|, \\
I_{L \rightarrow R}^{e}(\theta, \phi)=\frac{2 e}{h} \int_{-|\Delta(-\theta)|}^{-|\Delta(\theta)|} \frac{1}{\left|u(\theta)^{2}-v(\theta)^{2}\right|} \\
\times\left[|C(\theta, \phi)|^{2}-\frac{|D(\theta, \phi)|^{2}}{\left|u(\theta)^{2}\right|+\left|v(\theta)^{2}\right|}\right] d \epsilon \\
\quad \text { for }-|\Delta(-\theta)|<\epsilon<-|\Delta(\theta)|, \quad \text { (30) }
\end{gathered}
$$

where the factor $1 /\left|u(\theta)^{2}\right|+\left|v(\theta)^{2}\right|$ is used to normalize the wave function of leaving hole like quasiparticles. Considering that $\left|u(\theta)^{2}\right|+\left|v(\theta)^{2}\right|=1$, for $\epsilon<-|\Delta(\theta)|$, we write Eqs. (29) and (30) together in a singular expression

$$
\begin{aligned}
I_{L \rightarrow R}^{e}(\theta, \phi)= & \frac{2 e}{h} \int_{-\infty}^{-|\Delta(\theta)|} \frac{1}{\left|u(\theta)^{2}-v(\theta)^{2}\right|}\left[|C(\theta, \phi)|^{2}\right. \\
& \left.-\frac{|D(\theta, \phi)|^{2}}{\left|u(\theta)^{2}\right|+\left|v(\theta)^{2}\right|}\right] d \epsilon .
\end{aligned}
$$

Notice that when $u( \pm \theta)$ and $v( \pm \theta)$ are all real, from Eqs. (22) and (23), it can be easily found that $I_{L \rightarrow R}^{e}(\theta, \phi)$ equals to $I_{R \rightarrow L}^{e}(\theta, \phi)$, and the "pure" continuum with energy below $-\max (|\Delta(\theta)|,|\Delta(-\theta)|)$ makes no contribution to the total current. Then for the point contact considered, all the continuum currents are carried by the semileaky states, which develop from one branch of discrete Andreev levels with larger gap magnitude and the currents carried by them should be included in the discret currents in the $Z \rightarrow 0$ limit. The semileaky state would demonstrate itself as a supercurrent resonant peak, just as pictured in Fig. 7(b). The currents transported through these channels for two $Z^{*}$ values with $\alpha=20^{\circ}$ and $\theta=60^{\circ}$ are plotted. The solid line represents the conduction without quasiparticles crossing the Fermi surface ( $C$ process), corresponding to the contribution of $|C(\theta, \phi)|^{2}$, while the $D$ process associated with the process of free quasiparticles from one side turning into Cooper pairs on the other side shows a resonant structure (dashed lines) for a small $Z^{*}$ value 0.1 . When $Z^{*}$ is increased to 0.5 , the resonant peak drops and becomes cusplike as a result of the strong coupling of positive and negative processes and increasing quasiparticle lifetime. No such peak is found in the $C$ process. For small $Z$ values, the expression $C(\theta, \phi)$ can be expanded to the first order of $Z$,

$$
\begin{aligned}
C(\theta, \phi)= & \left(1+i Z^{*}\right) e^{-i \phi / 2}\left[1-v(\theta)^{2} / u(\theta)^{2}\right] /[1 \\
& \left.-e^{i \phi} v(\theta)^{2} / u(\theta)^{2}\right]+O\left(Z^{* 2}\right) .
\end{aligned}
$$

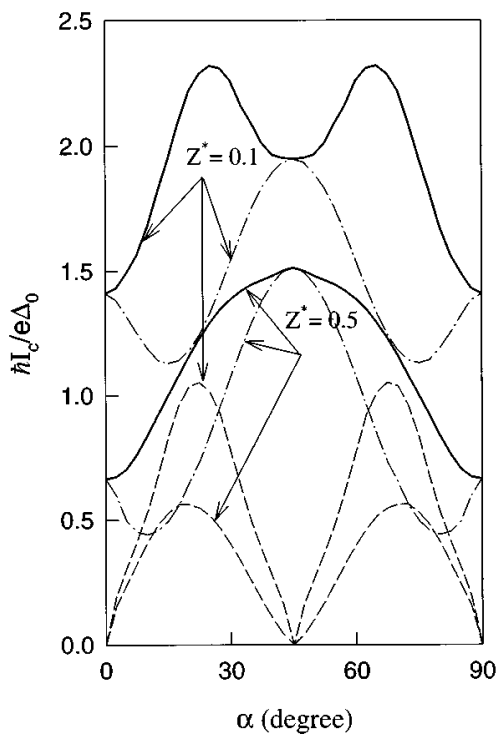

FIG. 8. Critical currents as a function of the tilt angle in the case of the DID configuration. Dashed lines and dot-dashed lines represent the contribution of quasibound levels and discrete Andreev levels, respectively.

In the intermediate-energy range, no net equilibrium current flow can be inferred from this form of $\phi$ dependent on $C(\theta, \phi)$. Our conclusion is that a considerable portion of the supercurrent is conducted across the Fermi surface, which is always negligible in $S N S$ junction configurations. $^{20,21}$ Regardless of existing normal scattering at the interface, the supercurrent jump at phase difference $\pi$ persists, while it is smoothed by the barrier in the $s$-wave Josephson junctions. The total discrete current and continuum (semibound) supercurrent for the DID junction with $Z^{*}=0.5$ and $\alpha=20^{\circ}$ are shown in the inset of Fig. 7(b). The phase dependence of their sum is somehow similar to the character of the extreme asymmetric ballistic $S_{1} N S_{2}$ superconducting point contact in Fig. 3 of Ref. 20, where a strong asymmetry with $\Delta_{1} / \Delta_{2}=200$ is assumed.

We now investigate how the barrier potential can affect the dependence of the critical current on the crystal orientation angle. In Fig. 8, by varying the grain boundary tilt angle $\alpha$ from $0^{\circ}$ to $90^{\circ}$, the critical currents of two typical $Z^{*}$ values, together with the maximums of the corresponding discrete current and continuum currents, are calculated. The variation of the critical currents shows quite a different tendency: For a stronger barrier at the interface, it is analogous to the long clean $D N D$ structure, but the cusp at $45^{\circ}$ is more evident, while for a weak barrier, two humps turn up and the maximum of the critical current is shifted to about $25^{\circ}$, leaving a dip at $45^{\circ}$. One can find that this novel peak is associated with the coexisting continuum current peak (dashed lines), which vanishes at $0^{\circ}$ and $45^{\circ}$ due to the coincidence of the magnitude of the order parameters $\Delta(\theta)$ and $\Delta(-\theta)$. The semileaky states carry the most prominent supercurrent near $25^{\circ}$ since $\Delta(\theta)$ and $\Delta(-\theta)$ differ most from each other near this tilt angle (dot-dashed lines). On the other hand, when $\Delta(\theta)$ and $\Delta(-\theta)$ are in opposite signs, midgaps are formed. The discrete currents being the highest at 110 direction can be understood by the fact that the maximum 
angle range with opposite signs of $\Delta(\theta)$ and $\Delta(-\theta)$ is reached near $45^{\circ}$. The competition of both components brings about distinctive features at two $Z^{*}$ values, which has no correspondence in the $s$-wave superconductor and could be utilized as a criterion of $d$-wave character in future experiments.

\section{SUMMARY AND REMARKS}

We have studied the Josephson effect between two $d$-wave superconductors with elastic barriers presented at interfaces. The Andreev interference in two limiting cases are addressed. When two $d$-wave superconductors are coupled by a finite width metallic normal conductor, in the absence of normal reflection, Andreev levels along different directions are formed independently. In the point-contact limit, the mixing of propagating modes by the scattering barrier at the interface is explored to a great extent. The Andreev levels take on striking different characteristics and consequently give contrasting supercurrent behavior. The transmission across the Fermi surface makes quasiparticles in one electrode turn into Cooper pairs in the other side. The variation of the critical current on the boundary angle is not only determined by the anisotropic nature of the $d$-wave parameter but also greatly influenced by its sign.

Very recently, Tanaka and Kashiwaya ${ }^{23}$ calculated the local density of states (LDOS) of quasiparticles near the inter- face of a DID junction. It is revealed that the LDOS strongly depends on the relative angle of boundary face and both crystals' orientation. The LDOS at zero energy show an anomalous dependence on the phase difference $\varphi$, which was thought to give a uncommon temperature dependence of Josephson currents.

Finally, we would like to point out that a cylindrical Fermi surface has been assumed throughout the whole work. In the tight-binding model of Bloch electrons in a square lattice at half-filling, most high- $T_{c}$ the superconductors bear a square Fermi surface. The summation of the supercurrent should be done on this quite complicated Fermi surface. Nevertheless, we believe that our simplified model gives a sensible description of the DND superconducting heterostructure composed of high- $T_{c}$ superconductors. The supercurrent dependence on the relative orientation of two $d$-wave superconductors is not discussed in this paper. When there is a misorientation angle between two superconductors, the Fermi velocities in one specific direction will be different; this mismatch effect may need to be carefully taken into account.

\section{ACKNOWLEDGMENTS}

This work was supported by a RGC grant of Hong Kong, Grant No. HKU262/95P, and a CRCG research grant at the University of Hong Kong.
${ }^{1}$ For a review, D.J. Scalapino, Phys. Rep. 250, 329 (1995); M. Sigrist and T.M. Rice, Rev. Mod. Phys. 67, 503 (1995), and reference therein.

${ }^{2}$ D.J. Van Harlingen, Rev. Mod. Phys. 67, 515 (1995).

${ }^{3}$ A.G. Sun, D.A. Gajewski, M.B. Maple, and R.C. Dynes, Phys. Rev. Lett. 72, 2267 (1994).

${ }^{4}$ P. Chaudhari and Shawn-Yu Lin, Phys. Rev. Lett. 72, 1084 (1994)

${ }^{5}$ D.A. Wollman, D.J. Van Harlingen, W.C. Lee, D.M. Ginsberg, and A.J. Leggett, Phys. Rev. Lett. 71, 2134 (1993); D.A. Wollman, D.J. Van Harlingen, J. Giapintzakis, and D.M. Ginsberg, ibid. 74, 797 (1995).

${ }^{6}$ A. Mathai, Y. Gim, R.C. Black, A. Amar, and F.C. Wellstood, Phys. Rev. Lett. 74, 4523 (1995).

${ }^{7}$ D.A. Brawner and H.R. Ott, Phys. Rev. B 50, 6530 (1994).

${ }^{8}$ C.C. Tsuei, J.R. Kirtley, M. Rupp, J.Z. Sun, A. Gupta, M.B. Ketchen, C.A. Wang, and Z.F. Ren, J.H. Wang, and M. Bhushan, Science 271, 329 (1996); J.R. Kirtley, C.C. Tsuei, J.Z. Sun, C.C. Chi, L.S. Yu-Jahnes, A. Gupta, M. Rupp, and M.B. Ketchen, Nature 373, 225 (1995).
${ }^{9}$ J.H. Miller, Jr., Q.Y. Ying, Z.G. Zou, N.Q. Fan, J.H. Xu, M.F. Davis, and J.C. Wolfe, Phys. Rev. Lett. 74, 2347 (1995).

${ }^{10}$ C.R. Hu, Phys. Rev. Lett. 72, 1526 (1994).

${ }^{11}$ J.H. Xu, J.H. Miller, and C.S. Ting, Phys. Rev. B 53, 3604 (1996).

${ }^{12}$ Y. Tanaka and S. Kashiwaya, Phys. Rev. Lett. 74, 3451 (1995).

${ }^{13}$ J-X. Zhu, Z.D. Wang, and H. X. Tang, Phys. Rev. B 54, 7354 (1996).

${ }^{14}$ Y. Tanaka, Phys. Rev. Lett. 72, 3871 (1994).

${ }^{15}$ G. Deutscher and R. Maynard, Europhys. Lett. 30, 361 (1995).

${ }^{16}$ M. Sigrist and T.M. Rice, J. Phys. Soc. Jpn. 61, 4283 (1992).

${ }^{17}$ P.F. Bagwell, Phys. Rev. B 46, 12573 (1992).

${ }^{18}$ H. Takayanagi, T. Akazaki, and J. Nitta, Phys. Rev. Lett. 75, 3533 (1995).

${ }^{19}$ G.E. Blonder and M. Tinkham, Phys. Rev. B 27, 112 (1983).

${ }^{20}$ Li-Fu Chang and P. F. Bagwell, Phys. Rev. B 22, 15853 (1994).

${ }^{21}$ M. Hurd and G. Wendin, Phys. Rev. B 51, 3754 (1995).

${ }^{22}$ J. Bardeen and J.L Johnson, Phys. Rev. B 5, 72 (1972).

${ }^{23}$ Y. Tanaka and S. Kashiwaya, Phys. Rev. B 53, 9371 (1996). 